\title{
Numerical simulation of wind effects: a probabilistic perspective
}

\author{
Ahsan Kareem \\ NatHaz Modeling Laboratory, University of Notre Dame, Notre Dame, IN 46556
}

\begin{abstract}
Numerical simulations of wind loads and their effects are critical in the design of structures to ensure their safety under winds. The simulations range from generation of time histories of wind velocity, pressure and force fluctuations to structural response and assessment of attendant functionality and safety under service and design loads, respectively. Typically these schemes employ Monte Carlo based approaches that encompass model based simulations or information derived from observed data. The scope of simulations spans uni-variate to multivariate processes; uni-dimensional to multi-dimensional fields; Gaussian to non-Gaussian; stationary to non-stationary; conditional and unconditional cases. In order to accomplish these tasks, methods based on the time, frequency, or time-frequency domains are employed. This paper summarizes a historical perspective, recent developments and future challenges. Also included in the discussion are computational tools employed for data analysis, response analysis and its management, and prediction. Examples are presented to illustrate some of the topics discussed.
\end{abstract}

KEYWORDS: Monte Carlo; Simulation; Stationary; Gaussian; Non-Gaussian; Non-Stationary; Transient Events, Time, Frequency, and Time-Frequency Domains.

\section{INTRODUCTION}

Simulation of wind related processes is essential for time-domain analysis of structures under wind loads, e.g., Long span bridges, offshore structures, or tall buildings. Besides numerical analysis, digitally simulated data is also needed to drive computer controlled test facilities involving any type of setup, e.g., multiple fans or controlled damping systems for mitigation of motion. Typically these simulations are based on models describing the process to be simulated which may be an exact theoretical model, phenomenological or empirical model or observed data. These schemes can be broadly categorized into two classes: (1) Methods based on the summation of trigonometric functions (wave superposition); (2) Methods based on convolution of white noise with a kernel function or integration of a differential equation driven by white noise (digital filtering). These techniques vary in their applicability, complexity, computer storage requirements and computing time.

Some of the key issues involving simulations surface from the need to simulate: a very large number of points such as along the span of a long span bridge, which requires appropriate modeling and inclusion of imperfect correlation of the random processes/fields; modulations in both time and frequency in the case of transient wind field of a downburst; non-Gaussian features observed in the pressure fluctuations under separated flow regions; conditional simulation of data in the presence of a limited measurements, or loss of measured data. In some cases, it may become important to include all these features in a single simulation. Hurricanes passage is a potential example of this since extreme, imperfectly correlated, non-Gaussian loads impinge on structures and where limited observations may be also be available.

Initially, the simulation techniques focused on the generation of uni-variate and onedimensional processes. The simulation of processes more than single variate, or dimension, was first addressed by Borgman (1969), Shinozuka (1971) and Shinozuka and Jan (1972). 
The simulations schemes are characterized as: Conditional or Un-Conditional; Gaussian or non-Gaussian; Stationary/Homogenous or non-Stationary/non-Homogeneous; Univariate/UniDimensional; Multi-Variate/Multi-Dimensional. The conditional simulations, or stochastic interpolation schemes, can be used to simulate, or interpolate, data in between measured locations. These schemes are discussed in the following sections.

\section{WAVE SUPERPOSITION (SPECTRAL) BASED SCHEMES}

\subsection{Spectral Representation Approach}

One of the traditional approaches for simulation is to utilize a superposition of trigonometric functions with random phase angles, which has been the most popular, perhaps due to its simplicity (Shinozuka, 1971). Using large numbers of terms in the summation, the contiguous target energy spectrum is approximated by its discrete form. The Gaussianity of the simulated data is established by virtue of the Central Limit Theorem. The process, in principle, is applicable to multi-variate/multi-dimensional processes/fields. However, this approach for a large number of variates becomes very computationally inefficient. In this context, the summation of trigonometric functions may be carried out by utilizing a fast Fourier transform (FFT). The use of FFT improves the computational efficiency drastically, but not without the expense of increased demand on computer storage. The level of difficulty increases significantly for the simulations that involve long-duration multi-variate or multi-dimensional processes (Samaras et al. 1985). Significant progress has been made over the last several decades in the simulation of uni- and multivariate, uni- and multi-dimensional, conditional and un-conditional processes based on wave superposition schemes (e.g., Shinozuka and Deodatis, 1991; Shinozuka et al., 1990; Li and Kareem, 1991, 1993, 1995; Grigoriou, 1993; Mann, 1998; Chen and Kareem, 2005, Carassale and Solari, 2006).

The Cholesky decomposition of the cross power spectral density matrix (XPSD) has been widely utilized in digital simulation of multi-variate and dimensional schemes (Shinozuka and Jan 1972). Li and Kareem (1989, 1993 and 1995) introduced the concept of "stochastic decomposition" of the XPSD matrix for the simulation of stationary random processes, which was further extended to the simulation of non-stationary processes ( $\mathrm{Li}$ and Kareem, 1991, 1997). Central to the stochastic decomposition is the decomposition of a correlated vector-valued random process into a set of vector-valued subprocesses, such that any two component processes from the same subprocess are statistically fully coherent, while any two component processes from different subprocesses are non-coherent. Therefore, the simulation of the parent process is simplified by the simulation of noncoherent subprocesses. The Schur decomposition is often more attractive than the Cholesky decomposition as the former permits a relatively small number of modes to be utilized in the simulation (Shinozuka et al. 1990, Di Paolo and Gullo 2001, Li and Kareem, 1993). Similar gains are also possible with Cholesky decomposition through truncation of terms (Li and Kareem, 1995).

Regardless of the attractiveness of the FFT approach, concerns remain regarding the limitations imposed on the large number of simulation locations and the length of time-series dictated by the computer memory available as noted in Samaras et al. 1985 and Li and Kareem, 1993 and recently Chen and Kareem, 2002. To address this shortcoming, Li and Kareem (1993) introduced a numerical simulation scheme that combines the advantages of the wave superposition techniques and digital filtering to simulate continuous long-duration multi-variate random processes. This approach offers the simple convenience of conventional FFT based schemes; however, it does not suffer from the drawbacks of the required large computer memory that, in the past, has precluded the generation of long duration time series utilizing FFT. Central to this approach is a 
simulation of a large number of time series segments by utilizing the FFT algorithm, which are subsequently synthesized by means of a digital filter to provide the desired duration of simulated processes. It offers computational efficiency, convenience, and robustness. This scheme has immediate applications to the simulation of real-time processes. Other measures are possible to reduce the computational effort for a large number of simulation locations, e.g., ignoring of higher modes in the case of Schur decomposition and reducing the order in the case of Cholesky decomposition. Additionally, decomposition at limited frequency locations and larger time steps may also reduce computational effort with subsequent interpolations. In order to facilitate the use of stochastic simulation in the design office, the NatHaz Modeling Laboratory has developed a web-based simulation portal, which would allow simulation of multi-variate Gaussian random processes with prescribed spectral characteristics. An example of the portal is give in Figure 1, which would be available soon at http://www.nd.edu/ nathaz (Kwon et al. 2005).

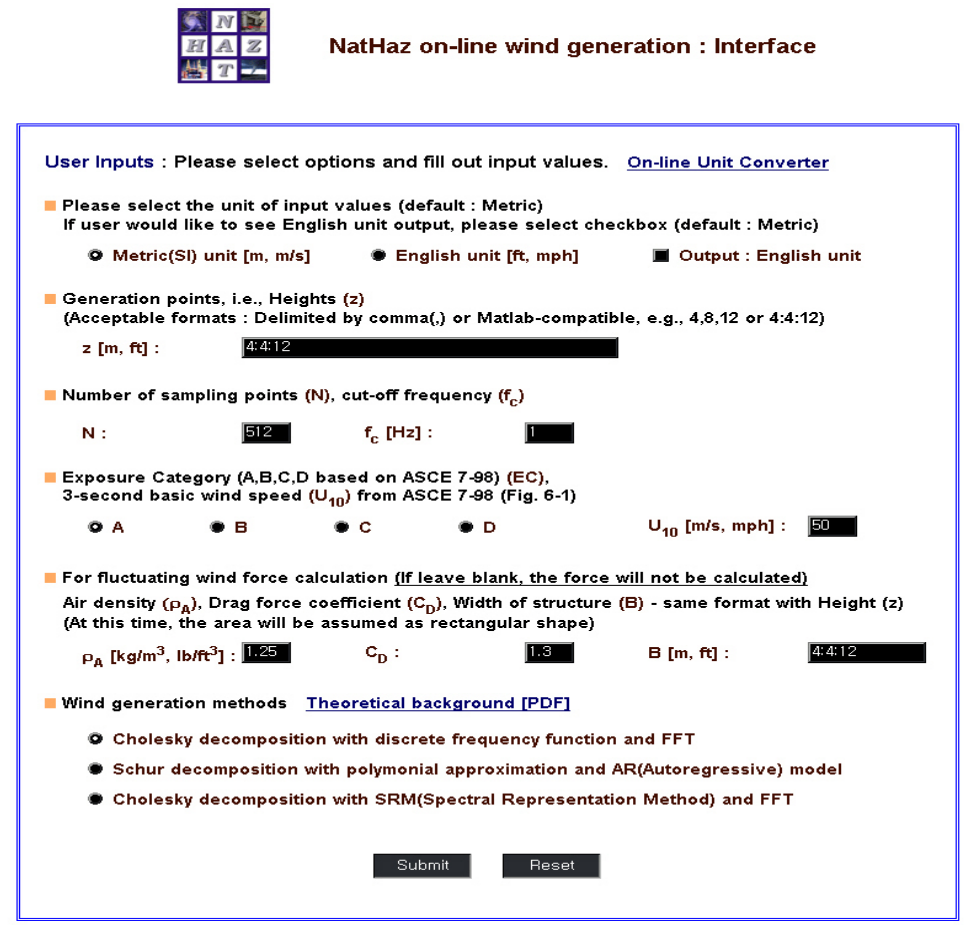

Figure 1: NatHaz Internet Simulation Portal

\section{DIGITAL FILTERING (TIME-SERIES APPROACH) BASED SCHEMES}

\subsection{Parametric Time-Series Methods}

The digital filtering based schemes offer efficient methods of simulating random processes. Typical schemes are: auto-regressive (AR), moving average (MA), and their combination autoregressive and moving averages (ARMA) (Samaras et al. 1985, Spanos and Mignolet 1992, Li and Kareem, 1989, 1990, 1991, 1993). The ARMA representation entails weighted recursive relations that connect the random quantity being simulated at successive time increments. Unlike the FFT-based approaches, this scheme does not demand large computer memory; rather, only a limited amount of information (e.g., coefficient matrices) is stored and long duration time series may be simulated at successive time increments. The matching of an ARMA model for certain 
applications may not be very straight forward, especially for those processes with filtered white noise as a spectral description. In lieu of this difficulty in selecting suitable model order, AasJakobsen and Strommen (2001) decided to use a spectral approach (Chen and Karem 2002b). The estimation of optimal model parameters is also sensitive to the time increment selected (Mignolet and Spanos, 1992 and Li and Kareem, 1990, 1993.

Based on the system's dynamic characteristics, the time-integration schemes require that the time increment should not exceed a prescribed value. This concern has been noted even in a recent study by Chay et al. (2006) dealing with the response of structures to downbursts where they resorted to linear interpolation, which introduces its own shortcomings. In order to efficiently simulate realizations of wind loads at the prescribed time increments, $\mathrm{Li}$ and Kareem (1990) offered two approaches. The first involved an ARMA algorithm based on a three-stagematching method and the other was based on a scheme which combines ARMA and a digital interpolation filter. This topic has been revisited by Wang and Kareem (2006) and offered an improved interpolation filter and demonstrated the shortcoming of the linear interpolation often used in practice.

The digital filtering approached can be directly implemented for multi- variate and dimensional processes/fields or they can be used in conjunction with a decomposition approach, Cholesky or Schur, in which the component processes are simulated as uni-variate processes. In Li and Kareem (1990) the dynamic response of structural systems to a variety of random excitations using recursive models was presented. The methodology permitted analysis of stationary, nonstationary, or transient response of structures under stochastic multi-input correlated processes, e.g., wind loads. For non-stationary or transient excitation, the analysis involved a direct computation of the output covariance from a given non-stationary correlation structure of the input. The concept of the ARMA system was also addressed in which the ARMA representation of the response was obtained in terms of the ARMA description of the stationary excitation. The usefulness of the recursive approach was also highlighted for nonlinear systems.

The time series approach also offers many useful recipes for numerical evaluations of commonly used operations. For example, discrete convolution models can be employed for linear transformation of a given time history, discrete differentiation models for obtaining derivatives, and discrete interpolation models for interpolating time series intermediate time increments and their hybrid combination ( $\mathrm{Li}$ and Kareem 1993). In a hybrid model, combined attractive features of the discrete convolution and differentiation models can be realized. This type of scheme is very effective in evaluating structural response with a displacement feedback, e.g., the response of a large floating platform exposed to ocean waves and winds which requires that the loads be formulated at the instantaneous displaced position of the platform ( $\mathrm{Li}$ and Kareem 1993).

\subsection{State Space Modeling}

In most modern control and dynamics studies, the state space is the format of choice. In this format, a state space model of the loading is needed which is augmented to the structural state space representation for the overall dynamic response analysis. Due to a large number of correlated wind-related processes with prescribed spectral characteristics, researchers have had difficulty in finding a state space model for wind (Gossman \& Waller 1983; Kareem 1999). Models for special applications have been tailored to meet problem-focused needs. One such approach involves a combination of stochastic decomposition and AR models to formulate a state space representation of wind effects (Kareem and Mei 1999). The XPSD matrix of wind-related process is decomposed utilizing Proper Orthogonal Decomposition (POD). Each component process is then modeled as an AR model, which is subsequently expressed in terms of state space format. By 
stacking these component models, the overall model for the entire system can be obtained in a straightforward manner. Details are available in Kareem and Mei (1999).

One of the most elegant applications of the state space model has recently presented in Chen and Kareem (2001) that addresses aeroelastic analysis of bridges under multi-correlated winds. An integrated state space model of a system with a vector-valued white noise input is presented to describe the dynamic response of bridges under the action of multi-correlated winds (Figure 2). Such a unified model has not been developed before due to a number of innate modeling difficulties. The integrated state space approach is realized based on the state space models of multi-correlated wind fluctuations, unsteady buffeting and self-excited aerodynamic forces, and bridge dynamics. Both the equations of motion at the full order in the physical coordinates and at the reduced-order in the generalized modal coordinates are presented. The state pace model allows direct evaluation of the covariance matrix of the response using the Lypunov equation, which presents higher computational efficiency than conventional spectral analysis approach. This state space model also adds time domain simulation of multi-correlated wind fluctuations, the associated unsteady frequency dependent aerodynamic forces and the attendant motion of the structure. It is important to note that frequency dependence of buffeting forces are maintained, unlike most time domain bridge aerodynamic studies which employ instead a straightforward quasi-steady approach. The structural and aerodynamic coupling effects among structural modes, which are critical for the multi-modal response of bridges, can be easily included in the analysis. The model also permits consideration of various nonlinearities, of both structural and aerodynamic origins, in the response analysis. Figure 3 shows an example simulation of wind fluctuations, buffeting, self-excited forces and bridge response.

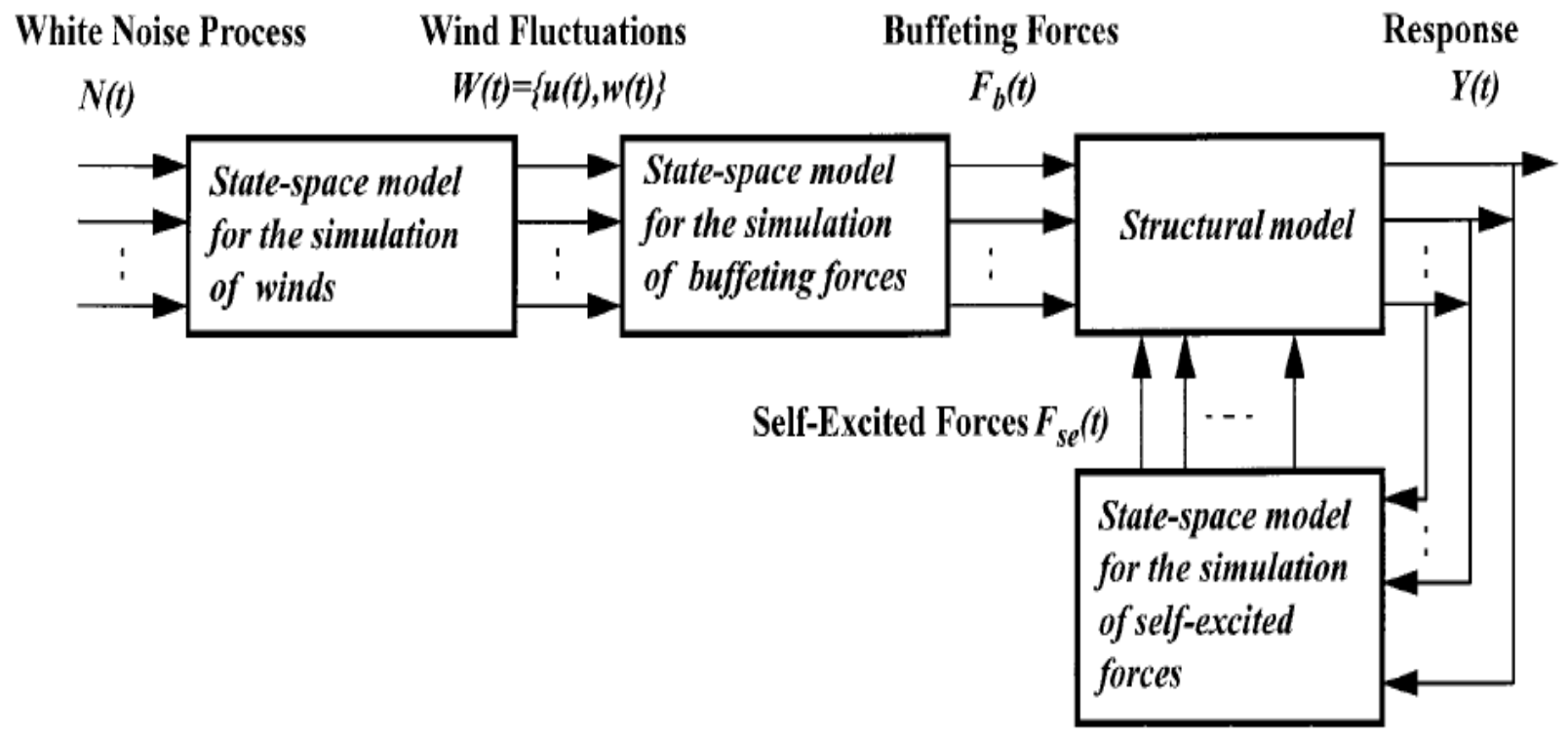

Figure 2: Integrated Modeling of Dynamic Response of Wind-Excited Structures (Chen and Kareem, 2005)

\section{SIMULATION OF INFLOW CONDITIONS}

Inflow conditions are important for developing large-eddy-simulations (LES) for modeling flow around an isolated structure or a group of buildings. In earlier studies, smooth uniform inflow conditions were introduced and combined with periodic boundary conditions to avoid difficulties 
associated with inflow and outflow conditions. For direct comparison with experimental results, it is highly desirable to perform simulations with inflow conditions which are comparable to those employed in experiments. One such approach is to utilize stochastic simulations of homogenous turbulence by statistical methods like those discussed here and in the preceding sections. Kondo et al. (1997) utilized the wave superposition approach in conjunction with FFT to simulate velocity fluctuations for LES simulation, which were then used by Tamura and Ono (2003) to study the influence of turbulence on the aerodynamics
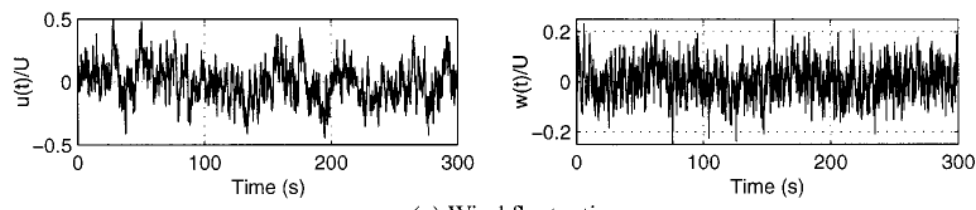

(a) Wind fluctuations
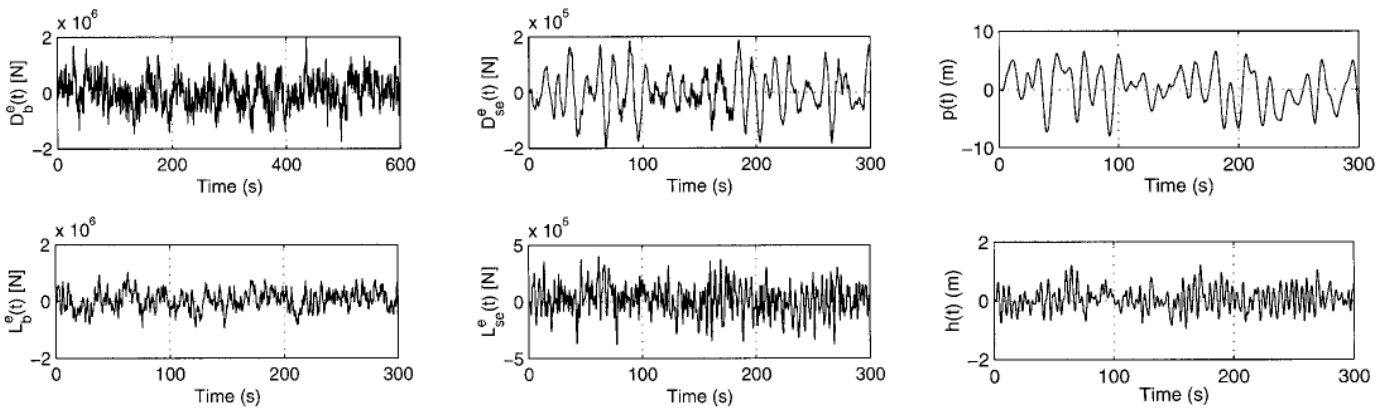

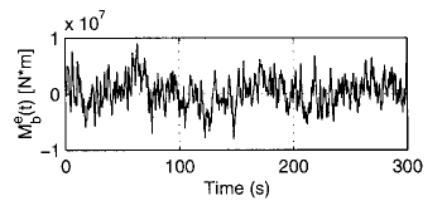

(b) Buffeting forces

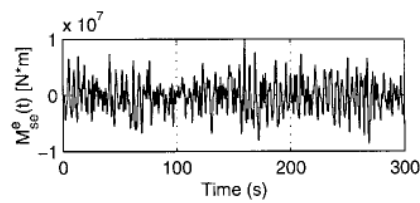

(c) Self-excited forces

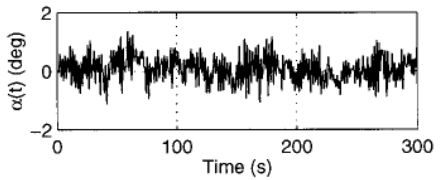

(d) Bridge response

Figure 3: Simulation of Wind Fluctuations, Buffeting and Self-Excited Forces, and Bridge Response (U=60m/s, at Midpoint of Main Span): a) Wind Fluctuations, b) Buffeting Forces, c) Self-Excited Forces, d) Bridge Response (Chen and Kareem, 2001).

of prisms. One of the issues for these simulations is to ensure that the flow is divergence free, which may require an iterative approach and should also consider the decay of turbulence as the flow moves into the computational domain. The addition of a boundary layer profile combined with turbulent fluctuations adds more complexity. More realistic inflow conditions can be simulated by generating flow over rectangular blocks whose size is adjusted based on the built conditions. This flow is then imposed on the computational domain for estimation of wind effects (Tamura et al. 2002). Other approaches deal with conditional simulations which are based on utilizing measurements of inflow turbulence in the wind tunnel and then conditionally simulating flow at all grind points as discussed in the section on conditional simulations.

\section{NON-STATIONARY/NON-HOMGENOUS ROCESSES}

The statistical description of severe wind events changes over the duration of the storm. The wind speed and turbulence intensity of hurricane and thunderstorm winds are in a constant flux 
as the storm passes over a fixed location. Wind models that account for this evolutionary behavior at multiple scales are just beginning to be realized. Non-stationarity has been particularly a difficult phenomenon to capture. Not only do the load statistics change with time and frequency, but the direction in which these loads impinge structures also changes, thus further complicating the load and performance assessment process.

Non-stationarity in wind storms stems from a number of origins, i.e., time varying mean, time varying variance and frequency dependent changes in variance. Typically, the time varying mean has been treated as a trend in the signal and, once removed, the remaining process is treated as stationary if other sources of non-stationarity are not present. The next level of non-stationarity stems from the time varying amplitude of the signal (Amplitude Modulation AM) which introduces time varying variance to fluctuations in wind. Further complications result from frequency modulations (FM) in which the variance varies with frequency contents. Finally, there are cases in which both AM and FM features are present.

Most of the simulations non-stationary processes have evolved around the generation of ground motion records known to be highly non-stationary. Following the introduction of the concept of evolutionary power spectrum (Priestly 1965), a variety of non-stationary stochastic models have been adopted to generate non-stationary ground motion records including the modulated filtered Poisson process (Shinozuka and Sato, 1967; Amin and Ang 1968), the modulated stationary process (Iyenger and Iyenger 1969) and filtered modulated stationary process (Levy et al. 1971). The development of non-stationary time-series models like AR, MA and ARMA has evolved over the years for the simulation of ground motion records (e.g., Cakmak et al. 1985; Deodatis and Shinozuka 1988). Despite the computational expediency, the time series based models for non-stationary processes may not be straightforward due to the elusive definition of the time-varying correlation and spectral function combined with the difficulty associated with the selection of the model order.

$\mathrm{Li}$ and Kareem (1991) introduced for the first time an FFT based simulation scheme for multivariate non-stationary processes. The utilization of FFT was made possible through the application of the stochastic decomposition advanced by the author and discussed earlier in this paper. A decomposed spectral matrix was expanded into a weighted summation of basic functions and time-dependent weights that are simulated by the FFT based algorithm. The effectiveness of the proposed technique was demonstrated using ground motion records that could be separated into time- and frequency-dependent components as well as for those in which both AM and FM modulations are present.

The addition of both AM and FM adds considerable complexity and temporal variation in frequency contents that can have a significant effect on structural response, especially in the inelastic range for earthquakes. This has made it critical to capture the transient features of both amplitude and frequency associated with earthquake, and potentially for wind, in order to make reliable predictions of structural response under extreme conditions.

In order to better model the temporal variations of frequency; estimates of evolutionary power spectral density have been made by decomposing a signal at various bands of frequencies using multiple band filtering (Kameda 1975, Scherer et al. 1982, Der Kiureghian and Crempien 1989). This can be viewed as the beginning of time-frequency analysis and modeling of transient signals. Further additions to this approach have been made based on short-time multiple window spectrum estimators in Conte and Peng (1997). These models have provided a sought out path for better modeling of temporal variations in both amplitude and frequency. Later, with the introduction of wavelets as a time-frequency analysis framework, researchers have explored it for the simulation of non-stationary processes (e.g., Gurley and Kareem, 1999). Empirical mode decomposition offers an alternate to wavelet based techniques, which has its advantages and short- 
coming in comparison with the wavelet transform based approaches (Kijewski-Correa and Kareem, 2006).

Nonstationary analysis of wind excited structures has been rather limited. Recently, interest in this area has developed in the aftermath of increasing damage to structures in extreme winds induced by tornados, thunderstorms winds and downbursts/microburst and hurricanes. The significance of transient wind events and their load effects can be readily surmised from an analysis of thunderstorm databases both in the U.S. and around the world, which suggest that these winds of gusts associated with convective gust-fronts differ significantly from conventional turbulence both in its kinematics and dynamics, e.g. their contrasting velocity profile and their transient nature. Accordingly, one should question the appropriateness of using design based on conventional represent the design wind speed for many locations. It is important to note that the mechanics with gusts associated with these transient events differ significantly from conventional turbulence both in its kinematics and dynamics, i.e., their contrasting velocity profile and their transient nature. Further, extreme loads on structures are potentially sensitive to the influence of transient flows, i.e., the load coefficients may be enhanced based on the gust form and the resulting localized, rapid changes in the surrounding flow. In addition, the characteristics of these flow structures suggest that the resultant load effects are likely to be correlated over larger areas than in conventional flows. These aerodynamic consequences clearly point at the prospect of higher loads on structures than would be predicted by the present codes and standards, calling for a careful examination of these procedures.

The recognition of the significance of wind loading on structures during the passage of thunderstorms has prompted research aimed at modeling and simulation of the downburst wind field based on measurements. However, the non-uniformity and non-stationarity of the wind field in downbursts in both time and space have posed challenges in their modeling and simulation. Wang and Kareem (2004 and 2005) have examined the evolutionary behavior of measured records of a thunderstorm outflow winds in a time-frequency framework involving the Wavelet and the Hilbert transforms. An efficient simulation model has been proposed on the basis that the instantaneous frequency of wind fluctuations at various frequency bands follows the Gaussian distribution. Without invoking an assumption associated with parametric models, this approach helps to characterize and simulate thunderstorm downburst winds based on a single sample measurement. Analytical expressions for the statistical properties of the underlying random processes are formulated. Examples concerning the simulation of actual full-scale downburst wind data are presented to demonstrate the efficacy of the proposed simulation that captures the evolutionary characteristics of measured data and preserves the correlation between measurements at different locations. In Figure 4, a sample of measured, time varying mean and simulated records are presented (Wang and Kareem, 2005). Verification of time histories with measured data was demonstrated by means of their time/frequency characteristics, e.g., co-scalograms.

Alternative models for downburst type winds have been obtained using a commercial software packages, e.g., FLUENT (Kim et al., 2005 and Chay et al. 2006), and based on amplitude modulated processes, which capture the time varying mean utilizing wavelet shrinkage and amplitude modulated fluctuations based on a separable time and frequency features (Chen and Letchford 2005 and 2005a). Other attempts have relied on the use of time varying ARMA models for the simulation of non-stationary features of downburst type winds (Chay et al, 2006). Similar type of models have been advanced for capturing the non-stationary features of hurricanes (e.g., Wang and Kareem, 2005; Xu and Chen, 2004). 


\section{NON-GAUSSIAN SIMULATION}

Significant progress has been made over the last decade in the modeling and simulation of Gaussian scalar, multi-variate and multi-dimensional conditional or unconditional random processes, but work in the area of non-Gaussian processes has been rather elusive (Yamazaki and Shinozuka 1988, Grigoriu 1994, 1998). The most commonly used approach to the simulation of nonGaussian processes is based on the static transformation that relates the underlying Gaussian process to the non-Gaussian process being simulated. This approach does not have any memory, whereas, schemes based on transformations with memory retain this feature, but there is very limited progress in this direction.

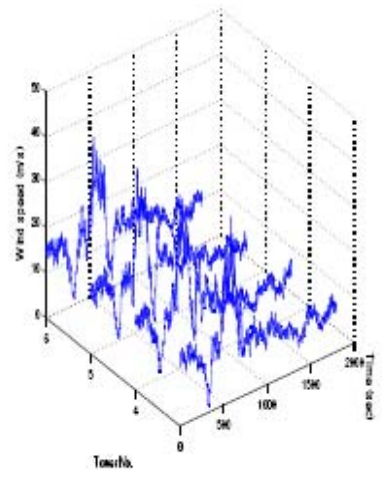

a) Measurements

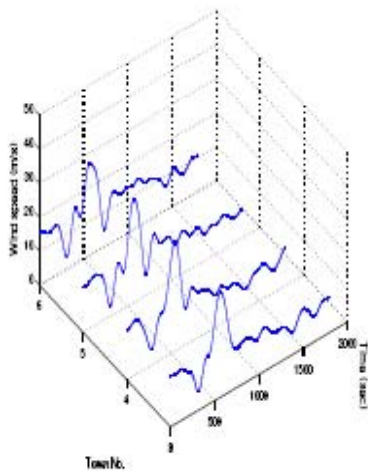

b) Time-dependent means

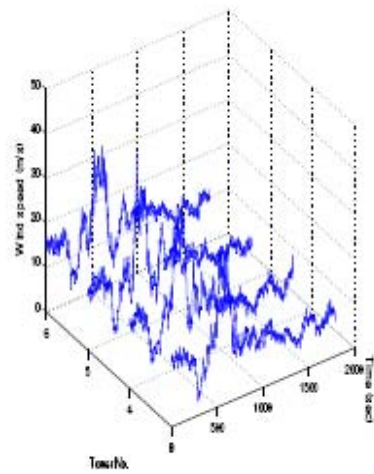

c) Simulations

Figure 4: Example Simulation of Full-Scale Downburst Winds (Wang and Kareem 2005).

\subsection{Static Transformation Schemes}

Early work based on correlation distortion has been based on an inverse mapping of the desired probability density function, a summary of which may be found in Gurley et al. (1996). In Gurley and Kareem (1997), a simulation approach, which is significantly more robust than the correlation distortion schemes, was presented. The non-Gaussian features and the frequency contents in the form of the first four moments and the target power spectral density of the process were used; alternatively analytical expressions or other estimates of the distribution may be employed. The approach is named the "Spectral Correction Method" and it relies on a few iterations to match both the spectral and probabilistic features.

Figure 5 shows an example of a simulation run based on a measured time history of a highly skewed full-scale pressure measurement. Also included are the probability density function (PDF) and power spectral density function (PSD) of the target and the simulated records which show good agreement. Additional follow-up work in this broad framework can be found in Grigoriu (1998), Deodatis and Micaletti (2001) and Masters and Gurley (2003). This approach has been extended to multi-variate processes, including conditional simulations and random fields (Gurley and Kareem, 1998; 1998a; Gurley et al. 2001). 


\subsection{Transformation Schemes with Memory}

Inasmuch as the preceding memoryless techniques are based on static transformations, they may fail to encapsulate any memory that may be present in the target signals. A Volterra series-based scheme offers an alternative that ensures preservation of memory. The Volterra based models in the frequency domain lend themselves to the simulation of non-Gaussian wind-related processes for which the Volterra kernels are available or estimated (Gurley et al. 1997, Kareem et al. 1998). The challenge remains in that the development of Volterra kernels needed for such simulation may not always be available (Gurley and Kareem, 1997).

\subsection{Other Approaches}

A class of single point non-Gaussian processes, e.g., pressure fluctuations at a location, can also be simulated through manipulation of the phase (Seong and Peterka, 1998). Gurley et al. (1996) utilized Neural networks to simulate non-Gaussian pressure time histories and demonstrated that due to the static nature of the approach, the simulated time history did not reflect the memory in the process as evidenced by the lack of good comparison with the target bi-spectra and the bispectra of the simulated record using Volterra kernel of Neural networks (Gurley et al. 1996 and 1997).

Future challenges in this area lie in the simulation of large vectors of data, which may be used in a design database, and in simulations that preserve memory inherent to the process. In the former case some space reduction techniques combined with linear stochastic estimation may be invoked to accomplish the task of simulating very large correlated data sets.
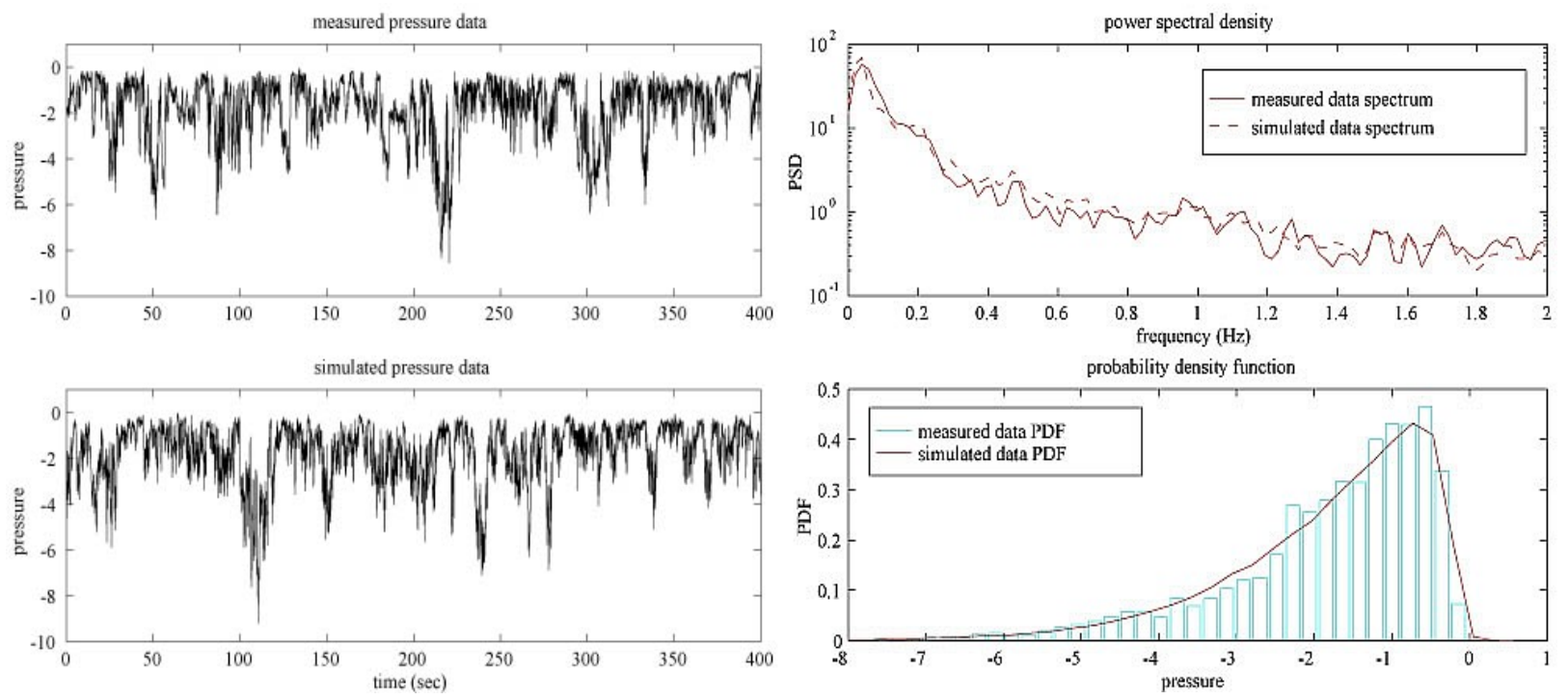

Figure 5: Measured and Simulated Pressure Time Histories (left); Comparison of PSD and PDF of Simulated and Measured Time Histories (right) (after Gurley, et al. 1997).

\section{CONDITIONAL SIMULATIONS}

\subsection{Gaussian Processes}

Simulation of random velocity and pressure time series at un-instrumented locations conditioned on the measured records are often needed in evaluating wind related studies. For example, mal- 
functioning instruments may leave a hole in a data set or information may be lacking due to limited number of sensors. The concept is similar to conditional sampling in experiments or numerical simulations. Fundamentally, two approaches have been introduced and the simulation is either based on linear estimation or kriging, or on a conditional probability density function (Krige, 1966; Borgman, 1990; Kameda and Morikawa, 1991). These approaches, being equivalent for uni-variate dimensional processes (Shinozuka and Zhang 1996), permit generation of time histories at new locations when one or more time series for the full length interval are available, and extension of existing records beyond the sampling time for cases where conditioning time series is limited to small subintervals. Gurley et al. (1997) have utilized the conditional probability density function approach to simulate one of the measured velocity records based on the availability of the other three on a full-scale tower. Figure 6 shows these records including the conditionally simulated. The comparison of the co-spectra between the simulated and measured records and the power spectral density, though not shown here for brevity, provides good agreement. In Figure 7, a measured record up to 2500 seconds shown in the top figure is assumed to be only available up to 980 seconds, indicated by the darkened portion of the record. The remaining record from 980-2500 seconds is simulated, as shown in lighter color in the bottom figure, based on the information derived from the first 980 seconds of data. Both examples demonstrate the effectiveness and utility of conditional simulation of Gaussian processes. An interesting application of this approach has been noted in developing inflow conditions for a computational fluid dynamics study in which measured turbulence at limited locations was expanded to the entire grid through conditional simulation (Muruyama and Morikawa 1995).

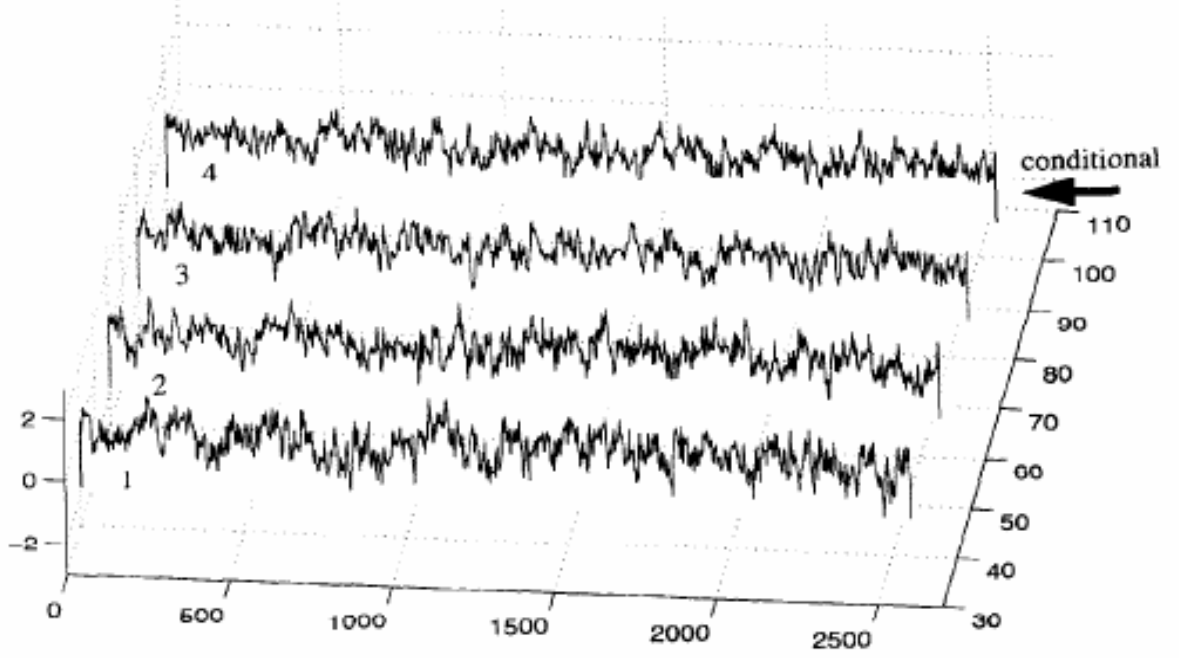

Figure 6: Example of Conditionally Simulated Time History (Gurley et al. 1997)

Similar schemes are possible using linear stochastic estimation (LSE) in which conditional information at reference points are used to estimate information over the entire flow domain (Adrian 1994). In this approach estimated values of the flow field at points conditioned on measurements at reference locations and time are expressed in terms of the Taylor's series expansion. By minimizing the mean quadratic error, involving cross-correlation, and retaining the first-order terms, desired estimates are obtained. Essentially, LSE estimates a random variable as a linear combination of some known reference variables. LSE has the ability to reconstruct the entire random filed by using a few reference variables in conjunction with attendant correlations. This approach can also help to couple experiments with CFD in which the experimental time histories, measured at limited locations, are interfaced with the inlet section of a time varying numeri- 
cal simulation of a spatially developing flow (Delville et al. 2000). Applications in wind engineering have been explored in Chen et al. (2003) in which LSE was utilized to reconstruct windinduced pressure time series from the covariance matrix for structural load analyses on a low building roof. It is a noted that this approach helps to reduce data storage for large scale databases, where one may reconstruct data based on stored covariance matrices and few reference measured pressures. It should be noted that the authors were unable to faithfully reproduce individual time series, but the integrated effect of several time histories resulting in a panel load were successfully replaced. This difficulty in matching individual time history may result from the non-Gaussian nature of the data.
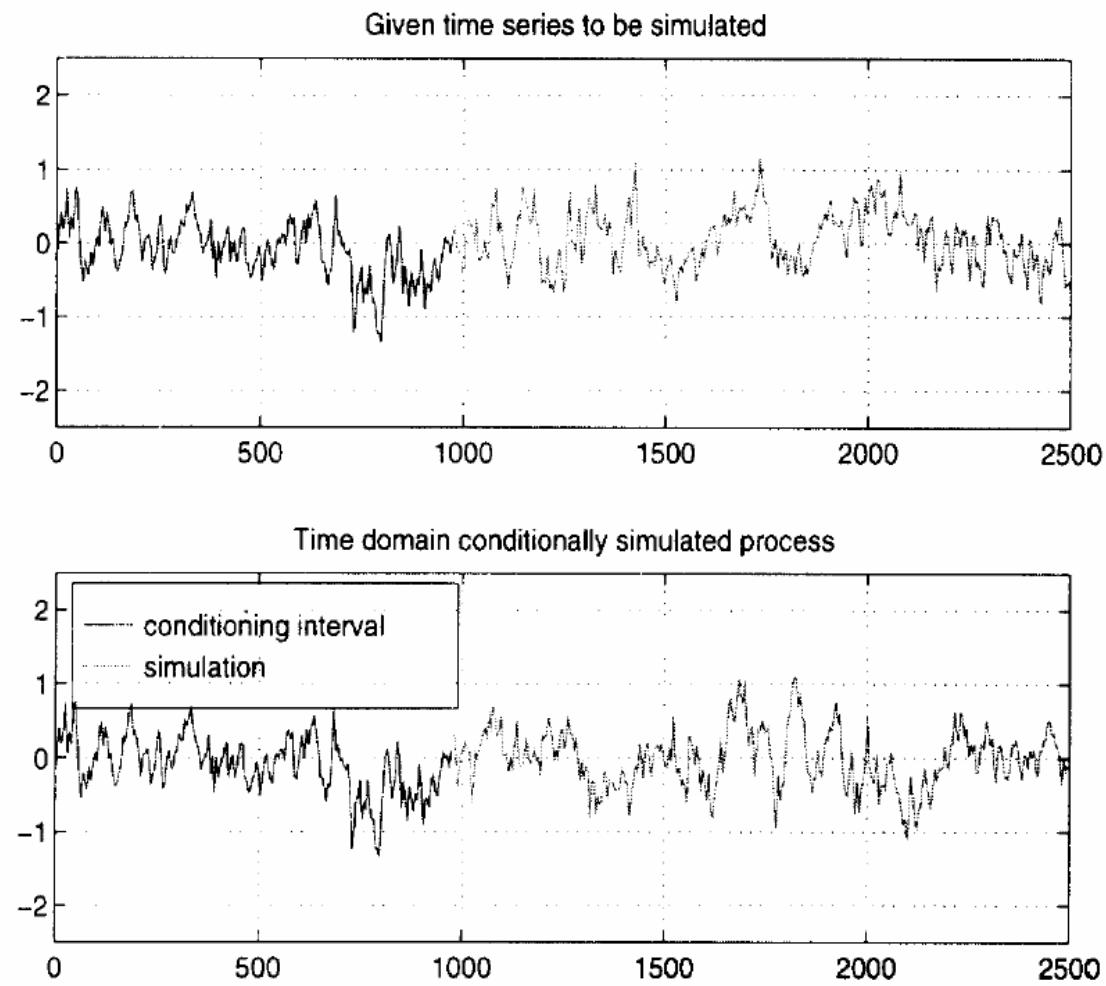

Figure 7: Example of a Simulated Time History Record (Gurley et al. 1997)

\subsection{Non-Gaussian Processes}

The preceding discussion was based on conditional simulations of Gaussian variates. Here, this concept is extended to non-Gaussian cases often experienced in wind engineering concerning pressure fluctuations in separated flow regions or velocity fluctuations in extreme atmospheric flow conditions. A non-Gaussian conditional simulation given in Elishakoff et al. (1994) addressed problems related to wide-band processes. In Gurley and Kareem (1998), a multi-variate non-Gaussian simulation based on a spectral correction scheme was extended to conditional simulation utilizing the conditional probability density function approach which is applicable to both narrow- and wide-banded processes. Details of the simulation scheme are available in Gurley and Kareem (1998). An example of the conditional simulation given in Gurley and Kareem (1998) involves pressure fluctuations given at three locations and at the fourth location the results we simulated. The left side of Figure 8 shows the three records and the simulated forth record. The right side of the figure shows the target and measured coherence between locations 1 
and 4, 2 and 4, and 3 and 4. The far right top in Figure 8 is a comparison of the PDF of the missing record and its replacement. This example and another dealing with extension of a single nonGaussian pressure record, not shown here, demonstrates that the conditional simulation scheme presented in Gurley and Kareem (1998) ensures excellent agreement between the target and simulated marginal distribution and spectral descriptions. Similar to the conditional simulation in Figure 6, extension of a missing record is shown in Figure 9, with a close-up of the transition from measured to simulated data location, in the bottom figure, to illustrate absence of discontinuity.
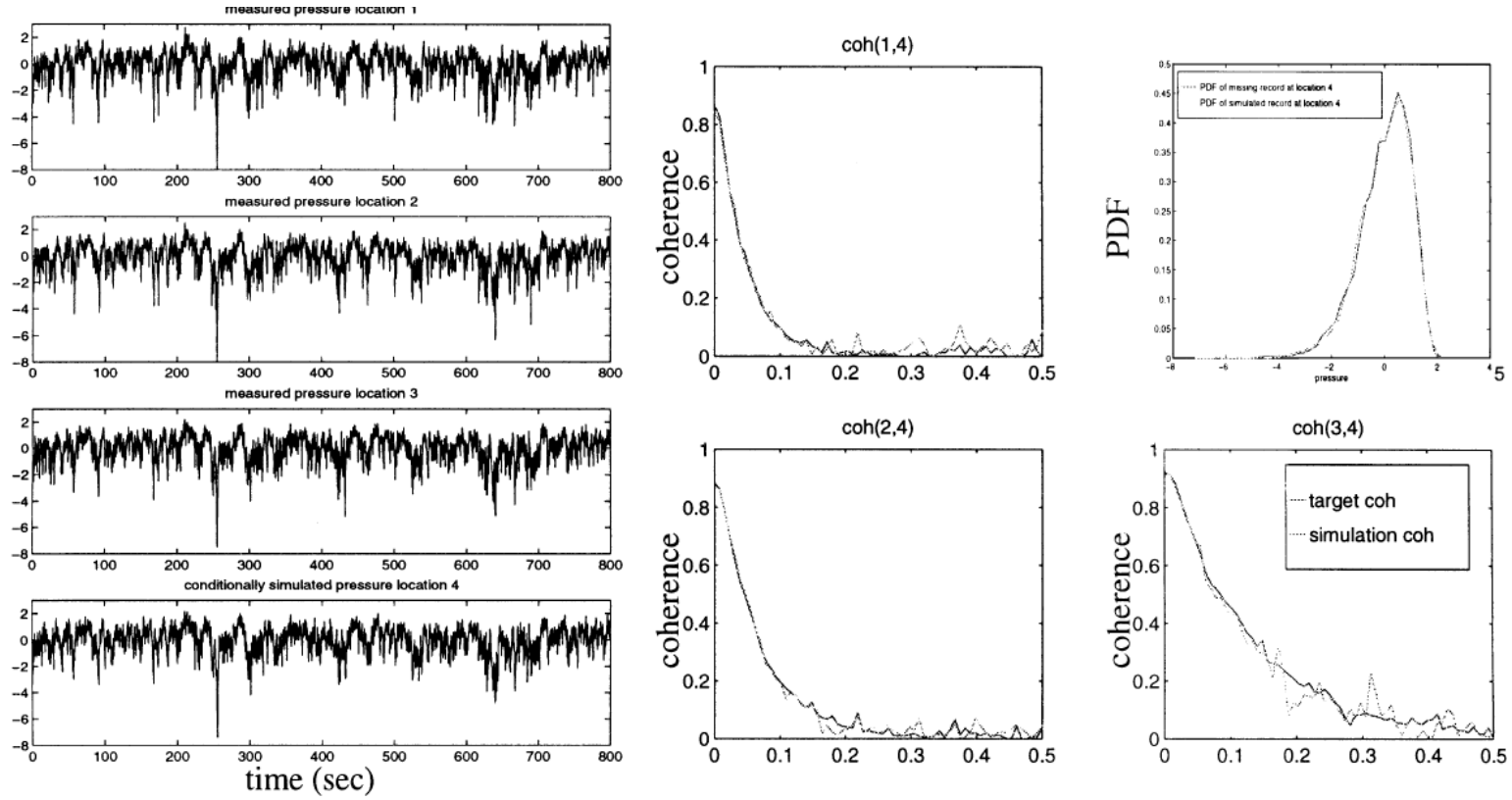

Figure 8: Example of a Simulated Signal for a Non-Gaussian Process (Gurley and Kareem 1998)

\subsection{Non-Stationary Processes}

In the previous two sections, discussion was focused on the conditional simulation of Gaussian and non-Gaussian vector-valued processes, which is extended to non-stationary vector valued processes. Application of the conditional simulation of ground motion records marked by nonstationary behavior are available in the literature, e.g., Vanmarcke and Fenton (1991), Hoshiya (1995), Shinozuka and Zhang (1996), Kameda and Morikawa (1994) and Heredia-Zavoni and Santa-Cruz (2000). These techniques are based on Kriging, conditional probability density function or their combination. Wang and Kareem (2006a) invoked the time-frequency framework to the conditional simulation of non-stationary. This scheme utilizes evolutionary cross-correlation in terms of cross wavelet coefficients, which extends the application of Kriging method to timefrequency domain. A numerical example of the conditional simulation of downburst wind velocities was presented to demonstrate the efficacy of the proposed scheme. Currently the scheme is being extended to conditionally simulate non-Gaussian and non-stationary processes.

\section{COMPUTATIONAL MODELING TOOLS}

\subsection{Proper Orthogonal Decomposition}

Multicorrelated stationary random processes/fields, such as wind velocity and pressure fluctuations, on structures can be transformed into a set of subprocesses by diagonalizing their covari- 
ance or cross power spectral density (XPSD) matrices through either the Cholesky (lower or upper triangular) or Schur (eigenvector) decomposition. The eigenvector decomposition offers physically meaningful insight into the process as each eigenvector (eigenmodes) may be characterized on the basis of its spatial distribution. It is also recognized that only a small number of eigenmodes corresponding to eigenvalues with larger magnitudes are dominant, such that one may ignore some of the eigenmodes associated with small eigenvalues in the description of a large-size random field. Accordingly, this technique provides a unique tool for data compression and facilitates a reduced-order modeling of large-size random fields. The eigenvector decomposition is theoretically based on the Karhunen-Loeve expansion, which is also known as the proper orthogonal decomposition (POD) (e.g., Loeve 1963).
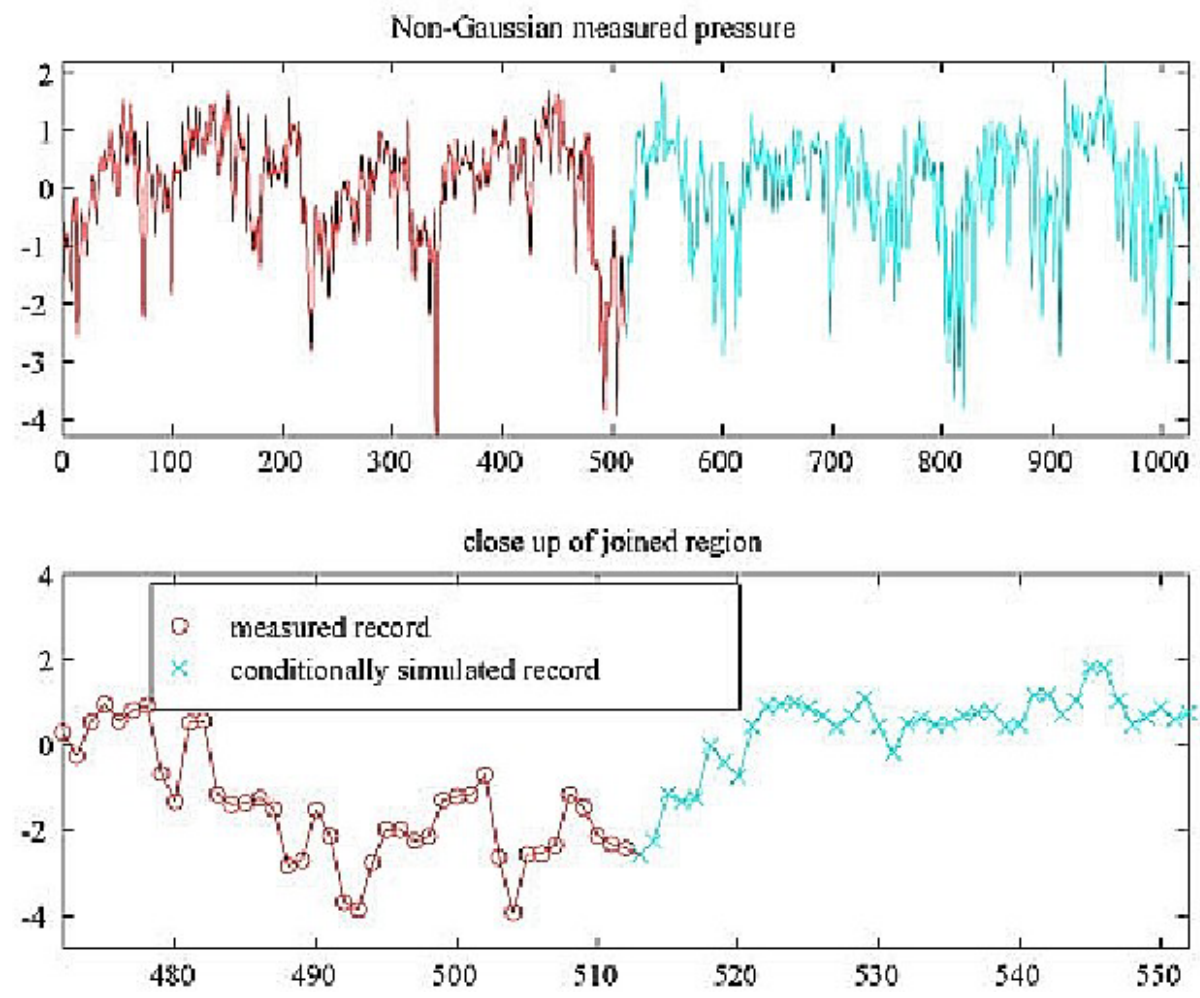

Figure 9: Example of Extended Data Record and Close-up of Extension Region (Gurley and Kareem, 1998)

The POD technique based on the covariance matrix has been widely used in many fields, such as fluid mechanics, image processing, signal analysis, data compression, and others. A comprehensive review on the subject is available in Solari et al. (2005). Lumley (1970) and Armitt (1968) introduced this technique to address turbulence and wind-related problems, respectively, and it was later used by many researchers in describing pressure fluctuations on buildings and structures and a host of wind-related problems (e.g., Lee 1975; Kareem and Cermak 1984; Holmes 1992; Kareem 1999; Tamura et al. 1999; Carassale et al. 2001). In stochastic structural mechanics, the POD technique based on the covariance matrix has been utilized for the simulation of spatially varying correlated random variables (e.g., Yamazaki and Shinozuka 1990), stochastic finite element analysis (e.g., Ghanem and Spanos 1991), and stochastic dynamics (Li and Kareem 1989, 1995, Vasta and Schueller 2000).

The numerical advantage of the POD technique, akin to the modal analysis in structural dynamics, relies on the reduced-order representation through truncation of the higher eigenmodes associated with small eigenvalues. This reduced-order representation, of course, must warrant 
that the important characteristics of the random field and related quantities remain unchanged, or the modification resulting from the approximate representation is acceptable. Several studies on the covariance matrix-based POD technique have demonstrated that truncating higher wind loading modes helps to expedite computations of global wind loads and their effects, e.g., Tamura et al. 1999; Chen and Kareem 2000. However, truncation of higher modes may not work effectively in the case of local response, which may lead to an underestimation of the local wind loads and their effects (Chen and Kareem 2005).

\subsection{Time-Frequency Representation and System Identification}

Like many physical processes of interest, wind effects manifest nonstationary and nonlinear features and their complete characterization may not be accomplished via Fourier transforms, necessitating a new analysis framework in the time-frequency domain. The dual nature of wavelet transforms, being a simultaneous transform in time and frequency, justifies its recent extension to the analysis of interest to civil engineering, adapting the transform to a number of situations where Fourier transforms (for frequency domain analysis) or Hilbert transforms (for time domain system identification) were traditionally used to define quantities of interest. When considering the time and frequency information in tandem, wavelets can be used to determine the times and frequencies at which signal energy content is strongest through examination of scalograms and co-scalograms (Gurley et al. 1997, Gurley and Kareem, 1999, Kareem and Kijewski, 2002). Figure 9 displays a co-scalogram comparison for full-scale pressure measured on a building and the two upstream wind velocity records: the first record monitored simultaneously as the pressure time history under consideration and the second from a different wind event. Figure 10 shows the scalogram and co-scalograms of wind pressure and the first wind velocity that are knowingly correlated. Note the pockets of white beyond $250 \mathrm{~s}$. revealing time-varying pockets of correlation in different frequency bands. This may be compared to a similar analysis for wind pressure and velocity that are known to be uncorrelated, for which no marked white pockets, indicative of correlation, are present. The presence of subtle light pockets, indicative of spurious correlation, present in both the correlated and uncorrelated examples in Figure 10 is due to lack of ensemble averaging (Kareem and Kijewski, 2002).

More specific insights into the linear and quadratic interplay between two signals in both time and frequency can be gained utilizing wavelet coherence and bi-coherence measures (Gurley et al. 2003). However, by exploiting the dual potential of wavelets, other analysis based primarily in either the time or frequency domain can also be performed. By tracking the variation of wavelet transform coefficients in the time domain, system identification can be readily performed (Kijewski and Kareem, 2003). Similarly, the distribution of wavelet coefficients with frequency at a window in time provides a familiar spectral representation whose evolutionary properties can be monitored to provide insights into nonlinear behavior (Kareem and Kijewski, 2002).

Recently, in order to capture intermittent correlation, a wavelet coherence measure was introduced to produce a time-frequency display of the coherence between signals intermittently correlated, e.g., pressure and velocity fluctuations (Kareem and Kijewski, 2002; Gurley et al. 2003). In this study, the analysis developed for first-order correlation detection was further extended to higher-order correlation through a bi-coherence measure, as some of the processes under investigation may not have been linearly related, e.g., wind velocity fluctuations and associated pressure fluctuations. Detailed examples given in (Kareem and Kijewski, 2002, Kijewski and Kareem, 2003) affirm that the wavelet-based technique is capable of identifying both first- and second-order correlation while effectively reducing the presence of noise in both simulated and measured data. The robustness of the provided thresholding techniques is further established, as it is shown to alleviate the presence of spurious coherence, even in cases where variance and 
leakage are prevalent. Though relatively intensive, this approach facilitates the removal of significant levels of all of the various contributing noise sources.

Wavelet-based system identification and instantaneous spectral analysis offer very valuable tools that permit the tracking of nonlinear characteristics in structural frequency and damping. Such tracking of time-varying frequency content is typically accomplished by monitoring the instantaneous frequency of the signal and extracting ridges from wavelet scalograms to form the Wavelet Instantaneous Frequency Spectra discussed in Kareem and Kijewski (2002) and Kijewski and Kareem (2003). The complex coefficients associated with these ridges can be used directly in a traditional system identification approach based on analytic signal theory to identify the instantaneous frequency, and in the case of free vibration decay or random decrement signatures, damping. Frequency domain perspectives from the wavelet coefficients are also insightful, as the wavelet instantaneous frequency and bandwidth can be respectively tracked to monitor the mean frequency and its deviation as they evolve in time. This basic analysis framework coupled with analysis schemes like SVD and PCA, would help to facilitate delineation of aerodynamic, aeroelastic or structural parameters.
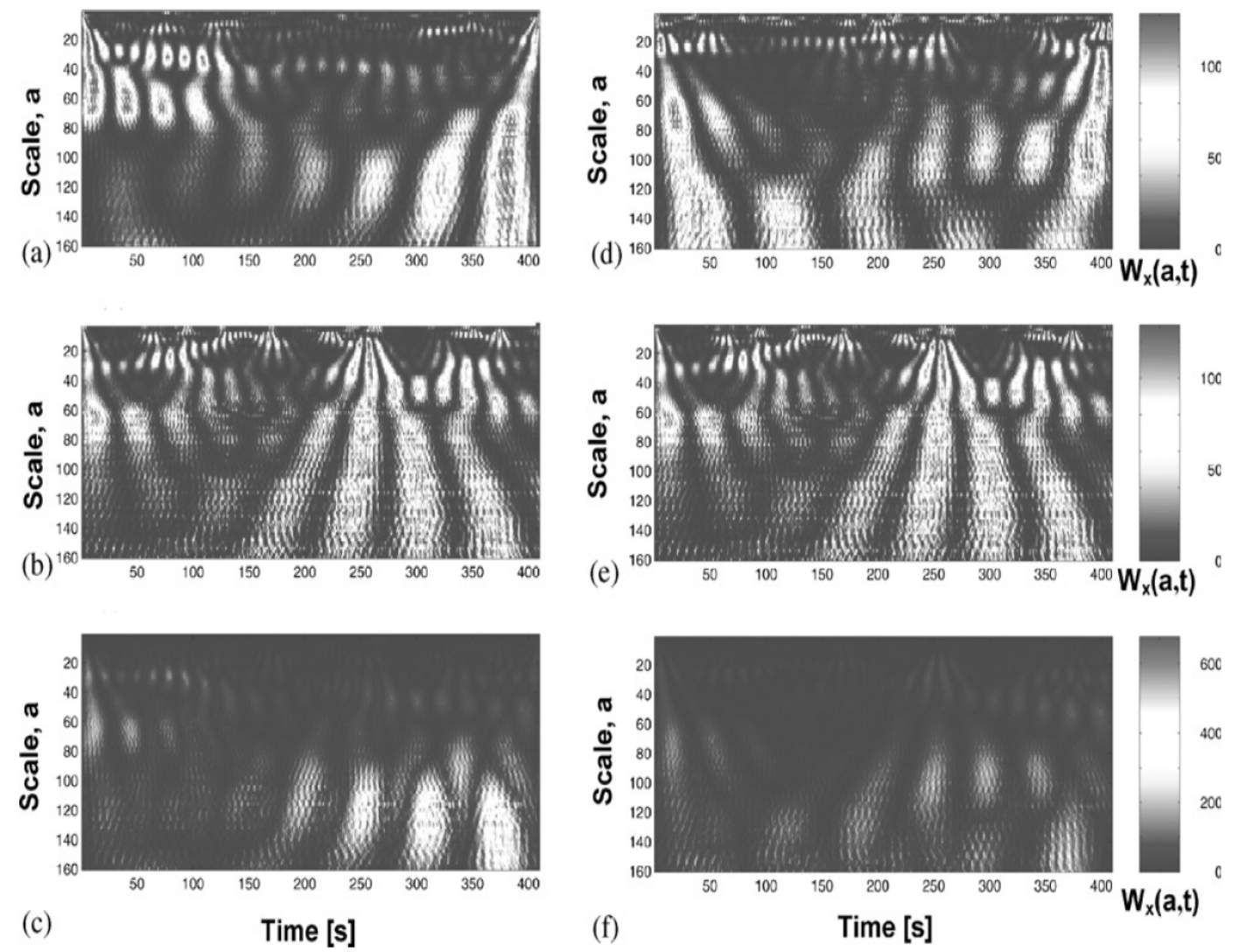

Figure 10: Example Wavelet Scalograms and Co-scalograms of Wind Pressure (Kareem and Kijewski 2002)

\section{TIME DOMAIN ANALYSIS OF STRUCTURES}

\subsection{Time Domain Approach for Coupled Flutter}

The prediction of wind induced buffeting response and flutter instability is of major concern in the design of long span bridges. The analytical approach has predominantly been conducted in 
the frequency domain. This is primarily due to computational efficiency offered by the frequency domain, particularly when handling the unsteady forces that are functions of frequency. The flutter analysis is generally conducted by complex eigenvalue analysis, whereas the buffeting response analysis is typically handled using a mode-by-mode approach that ignores the aerodynamic coupling among modes. More recently a coupled multi-mode flutter analysis framework has been introduced (Chen et al. 2000). This has led to a convenient transformation of the equations into a frequency independent state-space format. A significant feature of this approach is that an iterative solution for determining flutter conditions is unnecessary because the equations of motion are independent of frequency. In general, the frequency domain approach is restricted to linear structures excited by the stationary winds without aerodynamic or structural nonlinearities.

One of the challenges in aeroelastic analysis remains in the modeling of aerodynamic forces that take into consideration nonlinearities in both structural dynamics and aerodynamics and the ubiquitous issues related to turbulence (Chen and Kareem 2003, 2003a). Traditional analysis approaches are not suitable for accommodating these computational challenges. To include nonlinearities of structural and aerodynamic origins, the time domain approach is more appropriate (e.g., Chen et al. 2000 and the references cited). Most of the studies concerning the buffeting response have used the quasi-steady theory for modeling the aerodynamic forces, thus ignoring the frequency dependent characteristics of unsteady aerodynamic forces in the numerical scheme, potentially impacting the accuracy of the response estimates.

Time domain approaches also require input time histories of multi-dimensional multi-variate wind fields, which can be simulated as discussed in earlier sections on simulation. In Chen et al. (2000a), a comprehensive treatment of the time domain simulation of aerodynamic response of bridges was reported. Unsteady aerodynamic forces are expressed in terms of the convolution integrals involving the aerodynamic impulse functions and the structural motions or wind fluctuations. Subsequently, the aerodynamic impulse functions and the associated aerodynamic transfer functions (i.e., the flutter derivatives, admittance function and spanwise coherence) are approximated in terms of rational functions. A detailed parameter study was conducted in which the response of a bridge was modeled with and without modal coupling and the use of the quasi-steady aerodynamic force formulation for comparison with frequency dependent functions. For the case when buffeting forces are modeled by the quasi-steady theory, the forces are overestimated and hence the response is conservative. However, when the self-excited forces are also modeled by the quasi-steady theory, as most of the time-domain simulations have been, the aerodynamic damping will be overestimated and this leads to an underestimation of the response. For further details please refer the cited papers.

\subsection{Nonlinear Aerodynamic Response of Bridges}

Innovative aerodynamic tailoring of deck sections and advanced aeroelastic analysis frameworks are fundamental to the cost effective design of bridges with increasing complexity and span. Recent experiences have clearly demonstrated that traditional truss and box sections cannot be extended economically to longer spans. Further, the innovative sections with attractive aerodynamic performance, meant to take the place of more traditional sections, tend to exhibit aerodynamic nonlinearities. This situation is complicated by the fact that there is no conclusive evidence concerning the stabilizing or destabilizing effects of turbulence on the aerodynamics of bridges. While current analytical methods have proven their utility for past and current designs, their inability to fully analyze bridges with innovative sections and long spans, which are characterized by aerodynamic nonlinearities, turbulence effects and the spanwise coherence of aerodynamic forces, limits the utility of state-of-the-art analysis procedures, thus requiring a compre- 
hensive nonlinear analysis framework. The design of future, longer spans will require a multimode, coupled framework for flutter and buffeting analysis, which can accommodate the effects of turbulence, aerodynamic and structural nonlinearities, as discussed later in this section. Certainly, these analytical tools must be complemented by extensive wind tunnel and full-scale testing.

For many innovative bridge sections, even at low levels of turbulence, the effective angle of incidence due to structural motion and incoming wind fluctuations may vary to a level such that the nonlinearities in the aerodynamic forces may no longer be neglected. Wind tunnel studies using full-bridge, aeroelastic models have often shown flutter onset velocity boundaries to be sharply defined in smooth flows, whereas it exhibits rather gradual flutter boundaries in turbulent flows. A recent experimental study supports the full correlation of self-excited forces tacitly assumed in most current analytical approaches (Haan et al. 1999 and 2000). This implies that the turbulence-induced changes in flutter instability of bridges cannot be explained entirely due to a decrease in the coherence of self-excited forces, as suggested in Scanlan (1997). Therefore, one may conclude that current linear force models have proven their utility for a number of practical applications; however, these fail to completely address the challenges posed by aerodynamic nonlinearities and turbulence effects. Diana et al. (1999) proposed a nonlinear aerodynamic force model based on the so-called "Quasi-static corrected theory," which led them to analytically investigate the turbulence effects on flutter and buffeting response. This nonlinear force model attempted to incorporate frequency dependent characteristics by decomposing the total response into components with different frequencies. A recent study utilizing an active turbulence generator, demonstrated the validity of the approach in modeling bridge response to incoming turbulence (Diana, Bruni and Rocchi, 2005).

An advanced nonlinear aerodynamic force model and attendant analysis framework has been presented by Chen and Kareem (2003, 2003a) that focused on the needs for modeling of aerodynamic nonlinearity and effects of turbulence on long span bridges. The nonlinear force model separates the forces into the low- and high-frequency components in accordance with the effective angle of incidence corresponding to the frequencies lower than and higher than a critical frequency, e.g., the lowest natural frequency of the bridge. The low frequency force component can be modeled based on the quasi-steady theory due to its reduced velocity, while the highfrequency force component is separated into self-excited and buffeting components which are modeled in terms of the frequency dependent unsteady aerodynamic characteristics at the lowfrequency spatiotemporally varying effective angle of incidence. The nonlinear analysis framework is summarized in Figure 11 along with the conventional linear scheme. Within this framework, the effects of the low-frequency component of turbulence on flutter and buffeting responses can be analytically investigated. The effects of turbulence on flutter are modeled through the changes in the effective angle of incidence caused by turbulence and its influence on the self excited forces and the flutter instability.

The application of this framework to a long span suspension bridge, with aerodynamic characteristics sensitive to the angle of incidence, revealed a gradual growth in response with increasing wind velocity around the flutter onset velocity, which is similar to the wind tunnel observations of full-bridge, aeroelastic models in turbulent flows (Figure 12). This suggests that the effects of turbulence on the flutter of full-bridges may in part be attributed to aerodynamic nonlinearities, i.e., nonlinearities in the self-excited forces.

\subsection{Time Domain Response of Buildings}

Wind loads and the associated response of buildings have primarily been examined utilizing the high frequency base balance (HFBB) (Chen and Kareem, 2005, Zhou et al. 2003). The wind tun- 
nel based aerodynamic load data is utilized in conjunction with the building's computational model to estimate building response, which is then couched in a directional statistical description in terms of probability of exceedence of wind speed to arrive at the building's performance analysis for service and strength limit states. Typically these computations are done in the frequency domain due to it expediency. One can utilize the spectral description of aerodynamic loads derived from the HFBB and simulate a mode generalized time history of the corresponding load if it becomes necessary to use the time domain approach due to consideration of any nonlinear load effects. Additionally, multi-point synchronous measurements of pressure, which upon weighting with the tributary area of the respective tap, results in the overall integral loads as a function of time which can be interfaced with the appropriate structural model to derive time histories of response. The pressure approach offers more flexibility in terms of buildings with nonlinear mode shapes, and the complexity of model building these days can be avoided through computer controlled lithographic machines to construct complex model configurations with embedded pressure taps. Regardless, the HFBB has the advantage of permitting fast turn around in examining different external geometries at nominal cost for detuning the building shape to avoid adverse loading on the building, e.g., a case study reported in Abdelrazaq et al. (2005).

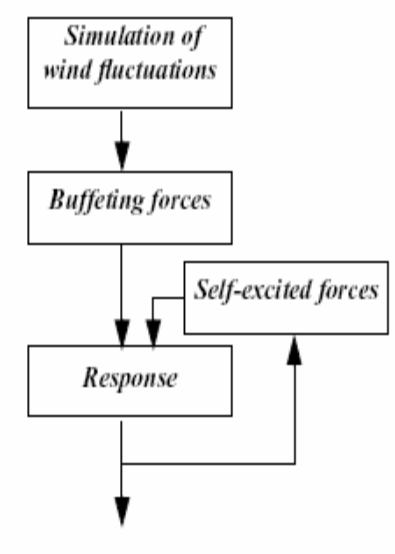

a) Traditional linear analysis framework

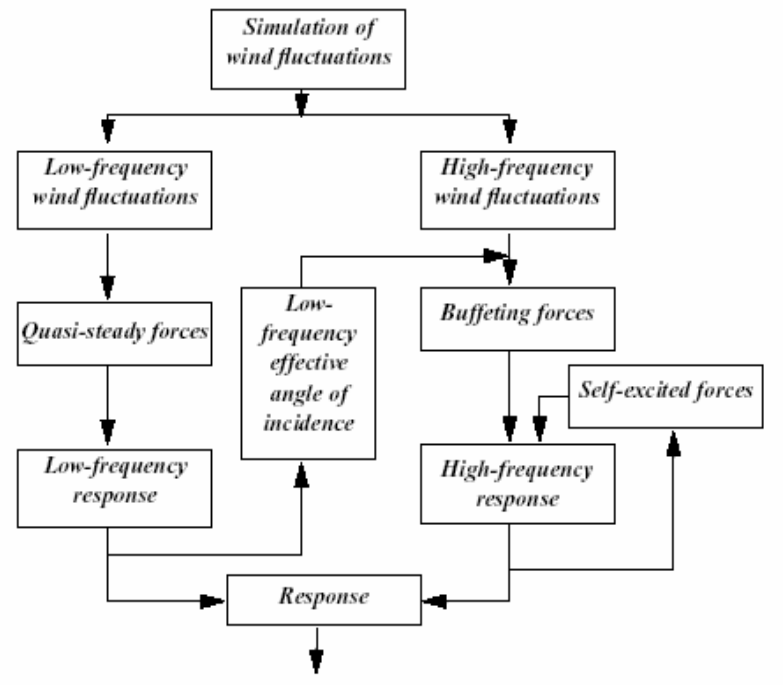

b) Proposed nonlinear analysis framework

Figure 11: a) Traditional and b) Nonlinear Aeroelastic Analysis Framework (Chen and Kareem, 2003a)
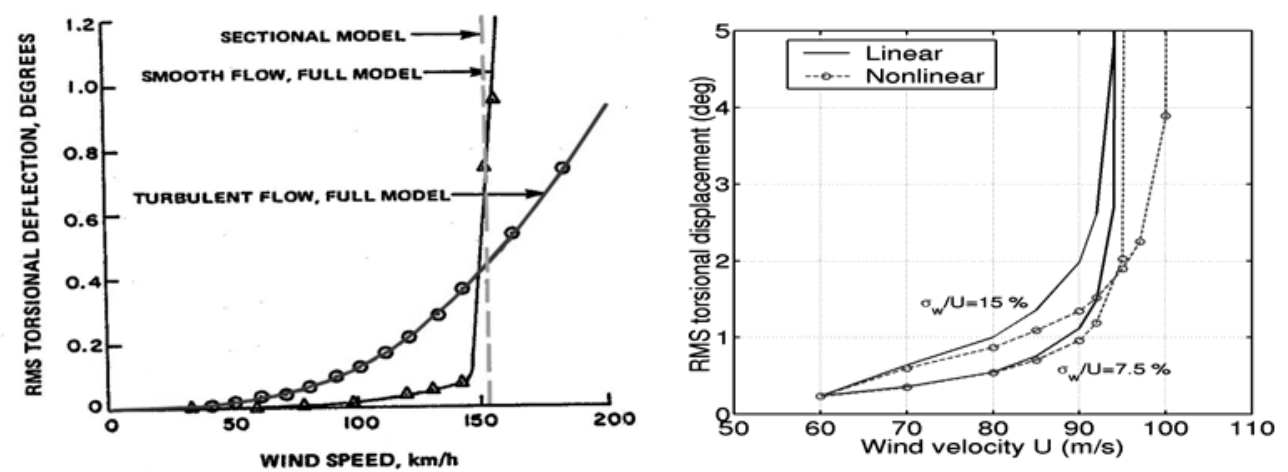

Figure 12: (Top) Torsional Response of Lions’ Gate Bridge (Irwin, 1977); (Bottom) Linear and Nonlinear Predicted Response of a Bridge (Chen and Kareem, 2003a). 


\section{CONCLUDING REMARKS}

This paper outlines developments in computational stochastic modeling of wind load effects beginning with some historical perspectives and discussing recent developments, improvements and challenges. The topics discussed include simulation of random processes that range from multi-variate to multi-dimensional cases involving Gaussian, non-Gaussian, stationary, nonstationary, conditional and un-conditional. These tools are becoming increasingly important in the analysis of wind excited structures that are exposed to a wide range of loads with attendant dynamic effects. Also discussed are customary time and frequency domain approaches and the emerging time-frequency analysis framework particularly important for non-stationary and nonhomogenous processes/fields. Applications of these computational techniques to the analysis of buildings and bridges are highlighted, including the role of stochastic simulation in providing inflow conditions for a computational wind engineering studies.

\section{ACKNOWLEDGEMENTS}

The support of the work reported has been made possible in part by the NSF grant CMS0324331 and earlier grants including other sponsors over the years. The author is indebted to the contributions of his former students and post-doctoral fellows on the subject of this paper. Kyle Butler and Rachel Bashor, current graduate students, are thanked for their assistance in the preparation of this manuscript.

\section{REFERENCES}

Aas-jakobson, K and Strommen, (2001), "Time domain buffeting response calculations of slender structures,” J. of Wind Eng. and Ind. Aerodyn, 89, 341-364.

Abdelrazak, A.K., Kijewski-Correa, T., Song, Y-H, Case, P., Isyumov, N. and Kareem, A., (2005), "Design and Full-Scale Monitoring of the Tallest Building in Korea: Tower palace III," Proc. of the Sixth Asia-Pacific Conference on Wind Engineering, Seoul, Korea, September, 2005.

Adrian, R. J. (1994), "Stochastic estimation of conditional structure: a review," App. Sci. Res., 53, 291303.

Amin, M. and Ang, A.H.S. (1968), “Nonstationary stochastic model of earthquake motions,” J. of Engrg. Mech., ASCE, 94(2), 559-583.

Armitt, J. (1968), "Eigenvector analysis of pressure fluctuations on the west instrumented cooling tower." Internal Rep. RD/L/N114/68, Central Electricity Research Laboratories, U.K.

Borgman, L.E. (1969), “Ocean wave simulation for engineering design,” J. Waterways and Harbor Div., ASCE, 557-583.

Borgman, L. E. (1990), “Irregular waves: kinematics and force,” The Sea, Prentice Hall, Englewood Cliffs, N.J.

Cakmak, A.S., Sherit, R.I. and Ellis, G. (1985), "Modeling earthquake ground motions in California using parametric time series methods," Soil Dynamics and Earthquake Engineering, 4(3), 124-131.

Carassale, L. (2005), "POD-Based filters for the representation of random loads on structures," Probab. Engrg. Mech., 20, 263-280.

Carassale, L. and Solari, G. (2006), "Monte Carlo simulation of wind velocity fields on complex structures,” J. of Wind Eng. and Ind. Aerodyn., 94, 323-339.

Carassale, L., Piccardo, G. and Solari, G. (2001), "Double modal transformation and wind engineering applications,” J. of Eng. Mech., ASCE, 127(5), 432-439. 
Chay, M.T., Albermani, F., Wison, R., (2006), "Numerical and analytical simulation of downburst wind loads," Engrg. Struct., 28, 240-254.

Chen, X., Matsumoto, M. and Kareem, A. (2000). "Time domain flutter and buffeting response analysis of bridges,” J. of Engrg. Mech., ASCE, 126, 7-16.

Chen, X., Matsumoto, M. and Kareem, A. (2000a). "Aerodynamic coupling effects on flutter and buffeting of bridges," J. of Engrg. Mech. ASCE, 126, 17-26.

Chen, X. and Kareem, A. (2001) "Aeroelastic Analysis of Bridges under Multi-Correlated Winds: Integrated State-Space Approach,” J. Engrg. Mech., ASCE, Vol. 127, No. 11.

Chen, X. and Kareem, A. (2002). “Advances in Modeling of Aerodynamic Forces on Bridge Decks,” $\underline{J}$. of Engrg. Mech., ASCE, Vol. 128, No. 11, pp. 1193-1205.

Chen, X. and Kareem, A., (2002a) "Advanced Analysis of Coupled Buffeting Response of Bridges: A Complex Model Decomposition Approach,” Prob. Engrg. Mech., Vol. 17, No. 2, pp. 201-213.

Chen, X. and Kareem, A., (2002b), "Discussion on time domain buffeting response calculations of slender structures by K. Aas-Jakobson and E. Strommen,” J. of Wind Eng. and Ind. Aerodyn, 90, 639642.

Chen, X., and Kareem, A. (2003). "New frontiers in aerodynamic tailoring of long span bridges: an advanced analysis framework," J. of Wind Eng. and Ind. Aerodyn., 91, 1511-1528.

Chen, X. and Kareem, A. (2003a). "Aeroelastic analysis of bridges: turbulence effects and aerodynamic nonlinearities,” ASCE J. of Engrg. Mech., 129(8).

Chen, X. and Kareem, A. (2005). "POD based modeling, analysis and simulation of dynamic loads," J. of Engrg. Mech., ASCE, Vol. 131, No. 4, pp. 325-339.

Chen, X. and Kareem, A. (2006). "Revisiting multimodal coupled bridge flutter: some new insights," ASCE J. of Engrg. Mech., in press.

Chen, L. and Letchford, C.W. (2005), "Proper orthogonal decomposition of two vertical profiles of fullscale nonstationary downburst wind speed," J. of Wind Eng. and Ind. Aerodyn., Vol. 93, No. 3., 187216.

Chen, L. and Letchford, C.W. (2005a), "Simulation of extreme winds from thunderstorm downbursts," Proceedings of the $10^{\text {th }}$ Americas Conference on Wind Engineering, June 2005, Baton Rouge.

Chen, Y., Kopp, G. A., Surry, D. (2003) "The use of linear stochastic estimation for the reduction of data in the NIST aerodynamic database," Wind \& Structures, 6(2), 107-126.

Conte, J.P., Peng, B.F. (1997), "Fully nonstationary analytical earthquake ground-motion model,” J., Engrg. Mech., 123(1), 15-24.

Delville, J., Lamballais, E. and Bonnet, J-P., (2000) "POD, LODS and LSE: their links to control and simulations of mixing layers," ERCOFTAC, Bulletin No. 46.

Deodatis, G. and Shinozuka, M. (1988), "Auto-regressive models for non-stationary stochastic processes,” J. of Engrg., Mech. ASCE, 114(11), 1995-2012.

Deodatis, G, Micaletti, R. C., (2001), "Simulation of highly skewed non-Gaussian stochastic processes," J. of Engrg. Mech., ASCE, Vol. 127, No. 12, 1284-1295.

Der Kiureghian, A. and Crempien, (1989). "An evolutionary model for earthquake ground motion," Struct. Safety, 6, 235-246.

Diana, G., Cheli, F., Zasso, A., Bocciolone, M., (1999), "Suspension bridge response to turbulent wind: Comparison of a new numerical simulation method results with full scale data," Wind Engineering into the 21st Century, Proceedings of the Tenth International Conference on Wind Engineering, Larsen et al. (eds.), Balkema, Rotterdam, p. 871-878.

Diana, G., Bruni, S. and Rocchi, D., (2005), "Experimental and numerical methodologies to define the response of a long span bridge to wind," Proc. of the Sixth Asia-Pacific Conference on Wind Engineering, Seoul, Korea, September, 2005.

Di Paolo, M., and Gullo, I. (2001). "Digital generation of multivariate wind field processes." Probab. Eng. Mech., 16, 1-10. 
Elishakoff, I., Ren, Y.J. and Shinozuka, M. (1994), "Conditional simulation of non-Gaussian random fileds,” Engrg. Struct., 16(7), 558-563.

Ghanem, R., and Spanos, P. (1991), Stochastic finite elements: A spectral approach, Springer, Berlin.

Grigoriu, M., (1984). “Crossings of non-Gaussian translation processes,” J. of Engrg. Mech., ASCE, Vol. 110, No. 4, 610-620.

Grigoriu, M., (1993), “On the spectral representation method in simulation,” Prob. Engrg. Mech., 8, 7590.

Grigoriu, M., (1998). "Simulation of stationary non-Gaussian translation processes” J. of Engrg. Mech., ASCE, Vol 124, No. (2), 121-126.

Gossman, E. and Waller, A. H., (1983). "Analysis of multicorrelated wind excited vibrations of structures using the covariance METHOD,” Engrg. Struct., 5, 264-272.

Gurley, K., Kareem, A. and Tognarelli, M.A. (1996). "Simulation of a class of non-normal random processes,” Journal of Nonlinear Mechanics, 31(5), 601-617.

Gurley, K. and Kareem, A. (1997). "Analysis, Interpretation, modeling and simulation of unsteady wind and pressure data," Journal of Wind Engineering and Industrial Aerodynamics, (67-71), 657-669.

Gurley, K., Tognarelli, M.A. and Kareem, A. (1997). "Analysis and Simulation Tools for Wind Engineering,” Probabilistic Engineering Mechanics, 12(1), 9-31.

Gurley, K. and Kareem, A. (1998). "A conditional simulation of non-normal velocity/pressure fields," Journal of Wind Engineering and Industrial Aerodynamics, (67-68), 673-684.

Gurley, K. and Kareem, A. (1998a). "Simulation of correlated non-Gaussian pressure fields," MECCANICA, 33(3), 309-317.

Gurley, K. and Kareem, A. (1999). "Applications of wavelet transforms in earthquake, wind and ocean engineering,” Engineering Structures, 21, 149-167.

Gurley, K., Jacobs, J. and Kareem, A. (2001). "Simulation of multidimensional non-Gaussian stochastic fields," Proceedings of the Monte Carlo Simulation, Schueller and Spanos, eds., Balkema, Rotterdam.

Gurley, K., Kijewski, T. and Kareem, A. (2003). "First and higher-order correlation detection using wavelet transforms,” ASCE Journal of Engineering Mechanics, 129(2), 188-201.

Haan, F.L., Kareem, A., Szewczyk, A.A., (1999), "Influence of turbulence on the self-excited forces on a rectangular cross section," Wind Engineering into the 21st Century, Proceedings of the Tenth International Conference on Wind Engineering, Larsen et al. (eds.), Balkema, Rotterdam, p. 1665-1672.

Haan, F.L., Kareem, A. and Szewczyk, A.A. (2000). "Experimental measurements of spanwise correlation of self-excited forces on a rectangular cross section," Volume of Abstracts, Fourth International Colloquium on Bluff Body Aerodynamics \& Applications (BBAA IV), Germany, 439-442.

Heredia-Zavoni, E. and Santa-Cruz, S. (2000), "Conditional simulation of a class of non-stationary spacetime random fields,” J. of Engrg. Mech., ASCE, 126(4), 398-404.

Holmes, J. D., Sankaran, R., Kwok, K. C. S., and Syme, M. J. (1997), "Eigenvector modes of fluctuating pressures on low-rise building models.” J. Wind. Eng. Ind. Aerodyn., 69-71, 697-707.

Hoshiya, M. (1995), "Kriging and conditional simulation of a Gaussian filed,” J. of Engrg. Mech., ASCE, 121(2), 181-186.

Irwin, P. (1977). Wind tunnel and analytical investigation of the response of Lions Gate Bridge to turbulent wind, NRC of Canada, NAE-LTR-LA-210.

Iyengar, R.N. and Iyengar, K.T.S.R. (1969). "A nonstationary random process model for earthquake accelerograms,” Bull.Seismological Soc. Of Am., 59(3), 1163-1188.

Kameda, H. (1975) "Evolutionary spectra of seismogram by multifilter," J. of Engrg. Mech., ASCE, 101(6), 787-801.

Kameda, H. and Morikawa, H. (1992), “An interpolating stochastic process for simulation of conditional random fields,” Prob. Engrg. Mech., 7(4), 242-254.

Kareem, A., and Cermak, J. E. (1984), "Pressure fluctuations on a square building model in boundarylayer flows.” J. Wind. Eng. Ind. Aerodyn., 16, 17-41. 
Kareem, A., Tognarelli, M. A. and Gurley, K. (1998), "Modeling \& analysis of quadratic term in the wind effects on structures,” J. of Wind Eng. and Ind. Aerodyn., (74-76), 1101-1110.

Kareem, A. (1999). Analysis and modeling of wind effects: numerical techniques, Wind Engineering into the $21^{\text {st }}$ Century, eds. Larson, Lindsey and Livesey, Balkema, Rotterdam.

Kareem, A., and Mei, G. (2000). "Stochastic decomposition for simulation and modal space reduction in wind induced dynamics of structures.” Applications of statistics and probability, R. E. Melchers and M. G. Stewart, eds., Balkema, Rotterdam, 757-764.

Kareem, A. and Kijewski, T. (2002). "Time-frequency analysis of wind effects on structures," Journal of Wind Engineering and Industrial Aerodynamics, 90, 1435-1452

Kijewski T. and Kareem, A. (2003). "Wavelet transforms for system identification: considerations for civil engineering applications," Computational Aided Civil and Infrastructure Engineering-An International Journal, 18, 341-357.

Kijewski-Correa and Kareem, A. (2006). "Efficacy of Hilbert and wavelet transform for the timefrequency analysis,” J. of Engrg. Mech., ASCE, 132(10), in press.

Kim, J., Ho, T.C.E. and Hangan, H. (2005), "Downburst induced dynamic responses of tall building," Proceedings of the $10^{\text {th }}$ Americas Conference on Wind Engineering, June 2005, Baton Rouge.

Kondo, K., Mochida, A and Murakami, S. (1997), "Generation of Velocity Fluctuation for Inflow Boundary Condition of LES,” Journal of Wind Engineering and Industrial Aerodynamics, 67-68.

Krige, D.G. (1966), "Two-dimensional weighted moving average trend surfaces for ore evaluation,” $J$. South Africa Inst. Of Mining and Metallurgy, Proc. Symp. On Math. Stat. and Comp. Appl. For Ore Evaluation, Johannesburg, South Africa, 1966. 13-38

Kwon, D., Kijewski-Correa, T. and Kareem, A. (2005), "e-Analysis/design of tall buildings subjected to wind loads," Proc. of the $10^{\text {th }}$ Americas Conference on Wind Engineering, Baton Rouge, LA.

Levy, R., Kozin, F., and Moorman, R.B.B. (1971), "Random processes for earthquake simulation,” J. of Engrg. Mech. ASCE, 97(2), 495-517.

Li, Y. and Kareem, A. (1989). "On stochastic decomposition and its application in probabilistic dynamics." Proc., $5^{\text {th }}$ Int. Conf., on Structural Safety and Reliability (ICOSSAR'89), A. H.-S. Ang, M. Shinozuka, and G. I. Schueller, eds., ASCE, New York.

Li, Y. and Kareem, A. (1990). “ARMA system in wind engineering.” Prob. Eng. Mech., 5(2), 50-59.

Li, Y. and Kareem, A. (1991). "Simulation of multi-variate nonstationary random processes by FFT," $J$. of Eng. Mech., ASCE, 117(5), 1037-1058.

Li, Y. and Kareem, A. (1993), "Simulation of multi-variate random processes: a hybrid DFT and digital filtering approach,” J. of Eng. Mech., ASCE, 119(5), 1037-1058.

Li, Y. and Kareem, A. (1995). "Stochastic decomposition and its applications to probabilistic dynamics," J. of Eng. Mech., ASCE, 121(1), 162-174.

Li, Y. and Kareem, A. (1997). "Simulation of multivariate nonstationary random processes: Hybrid DFT and digital filtering approach.” J. Eng. Mech., 123(12), 1302-1310.

Loeve, M. (1963), Probability theory, Van Nostrand, Princeton, N.J.

Lumley, J. L. (1970). Stochastic tools in turbulence, Academic, San Diego.

Maruyama, T. and Morikawa, H., (1995), "Digital simulation of wind fluctuation in a turbulent boundary layer using experimental data,” Proc. 9ICWE, New Delhi, I, 659-670.

Mann, J. (1999). “Wind Field Simulation,” Probabilistic Engineering Mechanics, 13(4), 269-282.

Masters, F. J. and Gurley, K. (2003), "Non-Gaussian simulation: Cumulative distribution function mapbased spectral correction,” J. of Eng. Mech., ASCE, 129(12), 1418-1428.

Mignolet, M.P. and Spanos, P.D. (1992). "Simulation of homogenous two dimensional random field"; Part I, AR and ARMA models”; Part II, J. of Applied Mechanics, ASME, 59, 260-277.

Priestly, M.B. (1965). "Evolutionary spectra and non-stationary processes," J. of the Royal Statistical Society Series B, 27, 204-237.

Samaras, E., Shinozuka, M., Tsurui, A. (1985), “ARMA representation of random processes,” J. Engrg. Mech., ASCE, 111(3), 449-461. 
Seong, S.H. and Peterka, J.A., (1998), "Digital generation of surface-pressure fluctuations with spiky features, Journal of Wind Engineering and Industrial Aerodynamics, 73, 181-192.

Scanlan, R.H. (1997). “Amplitude and turbulence effects on bridge flutter derivatives," ASCE Journal of Structural Engineering, 123(2), 232-236.

Sherer, R.J., Riera, J.D. and Schueller, G.I. (1982), "Estimation of the time-dependent frequency content of earthquake accelerations,' Nuclear Engrg. And Design, 71(3), 310-310.

Shinozuka, M. and Sato, Y. (1967), "Digital simulation of nonstationary random processes,” J. of Engrg. Mech., ASCE, 93(1), 11-40.

Shinozuka, M. (1971), "Simulation of multivariate and multidimensional random processes," J. of Acoustical Society of America, 49, 357-367.

Shinozuka, M. and Deodatis, G. (1991), "Simulation of stochastic processes by spectral representation," Appl. Mech. Rev.,44, 4, 191-203.

Shinozuka, M. and Jan, C. M. (1972). "Digital simulation of random processes and its applications.” J. Sound and Vib., 25(10), 111-128.

Shinozuka, M., Yun, C.-B., and Seya, H. (1990). "Stochastic methods in wind engineering." Journal of Wind Engineering and Industrial Aerodynamics, 36, 829-843.

Shinozuka, M. and Zhang, R. (1996), "Equivalence between Kriging and CPDF methods for conditional simulation,” J. Engrg. Mech., ASCE, 122(6), 530-538.

Solari, G. Carassale, L., Tubino, F. (2005), "POD methods and applications in wind engineering," Proc. of the Sixth Asia-Pacific Conf. APCWE-VI, Seoul, Korea, Sept. 2005.

Tamura, T. and Ono, Y. (2003), "LES Analysis on aeroelastic instability of prisms in turbulent flow," Journal of Wind Engineering and Industrial Aerodynamics, 91, 1827-1826.

Tamura, T., Okuda, Y. and Okada, H. (2002), "LES estimation of wind characteristics in the surface layer over various grounds - urban roughness effects and terrain effects," Proc. of the UJNR Panel on Wind and Seismic Effects (Task Committee D), (2002), Seattle.

Tamura, Y., Suganuma, S., Kikuchi, H., and Hibi, K. (1999), "Proper orthogonal decomposition of random wind pressure field.” J. Fluids Struct., 13, 1069-1095

Vanmarcke, E. H and Fenton, G.A. (1991), "Conditional simulation on local fields of earthquake ground motion,” Struct. Safety, 10(1-3), 247-263.

Vasta, M., and Schueller, G. I. (2000), "Phase space reduction in stochastic dynamics.” J. Eng. Mech., 126 61, 626-632.

Yamazaki, F. and Shinozuka, M. (1990). "Simulation of stochastic fields by statistical preconditioning", J. Eng. Mech., ASCE, 116(2), (1990), 268-287

Wang, L. and Kareem, A. (2004a) "Modeling of Non-Stationary Winds in Gust Fronts," Proc. of $9^{\text {th }}$ Joint Specialty Conference on Probabilistic Mechanics and Structural Reliability, Albuquerque, July.

Wang, L. and Kareem, A., (2005), "Modeling and simulation of transient winds: downbursts/hurricanes," Proc. of the $10^{\text {th }}$ American Conf. on Wind Engineering, Baton Rouge, LA, 2005.

Wang, L. and Kareem, A., (2006), "Interpolation Models,” in preparation.

Wang, L. and kareem, A., (2006a), "A Conditional simulation of downburst wind field,” in preparation.

$\mathrm{Xu}$, Y. L. and Chen, J., (2004). "Characterizing nonstationary wind speed using empirical mode decomposition,” J. of Struct. Engrg., ASCE, 130(6), 912-920.

Zhou, Y., Kijewski, T. and Kareem, A. (2003). "Aerodynamic loads on tall buildings: an interactive database,” ASCE Journal of Structural Engineering, 129(3), 394-404. 